results are limited by the small study sample size. They also stress that their results do not indicate that all HRT should be contraindicated for patients with partial epilepsy.

Original article Harden CL et al. (2006) Hormone replacement therapy in women with epilepsy: a randomized, double-blind, placebo-controlled study. Epilepsia [doi: 10.1111/j.1528-1167.2006.00507.x]

\section{Oxcarbazepine increases the levels of some androgens}

Oxcarbazepine is a relatively new antiepileptic drug with a similar efficacy to carbamazepine, but its effect on the reproductive endocrine function of women is unknown. Results of a recent study, however, indicate that oxcarbazepine is associated with an increase in the levels of some androgens and in the prevalence of polycystic ovaries.

Löfgren and colleagues from Finland recruited 35 women with epilepsy who were receiving monotherapy with either carbamazepine $(n=16)$ or oxcarbazepine $(n=19)$, and 36 healthy female controls. They carried out neurological and gynecological examinations of the participants, and collected blood samples during the early follicular phase of their menstrual cycles to analyze serum samples of reproductive hormones.

Although patients taking either carbamazepine or oxcarbazepine had lower serum levels of testosterone and free androgen than controls, patients taking oxcarbazepine were found to have higher serum levels of androstenedione and dehydroepiandrosterone sulfate than patients taking carbamazepine. There was also a higher prevalence of polycystic ovaries among patients receiving oxcarbazepine than in carbamazepine or control subjects.

The authors conclude that carbamazepine and oxcarbazepine appear to have differing effects on the reproductive endocrine function of women with epilepsy, and that oxcarbazepine could adversely affect women with epilepsy and hyperandrogenism. They add that further studies are needed to establish whether the endocrine changes are a result of the epilepsy itself or of the oxcarbazepine.

Original article Löfgren E et al. (2006) Effects of carbamazepine and oxcarbazepine on the reproductive endocrine function in women with epilepsy. Epilepsia [doi: 10.1111/j.1528-1167.2006.00506.x

\section{Human prion gene polymorphism influences Wilson's disease onset}

Studies indicate that the human prion-related protein (PrP) could be involved in copper homeostasis. A recent study in patients with Wilson's disease (WD) investigated whether a PrP gene polymorphism at codon 129-which results in the insertion of either methionine or valine at this position-affects the clinical phenotype of the disease.

Merle and colleagues recruited 134 patients with WD, of whom 46 had presented with neurological and 88 with hepatic symptoms. The researchers were primarily interested in whether the PrP M129V genotype affected the age of disease onset and its clinical expression (neurological or hepatic). Blood samples were collected, and genotype analysis carried out using polymerase chain reaction.

Overall, patients who were homozygous for methionine (PrP 129M/M) were found to be significantly older at the time of symptom onset than those who possessed at least one $V$ allele $(P=0.003)$. When patients with neurological symptoms were considered alone, age of symptom onset was also significantly higher in $\operatorname{PrP} M / M$ patients than in patients with at least one $\mathrm{V}$ allele $(P=0.01)$. There was no significant association between PrP genotype and type of clinical manifestation of disease.

The authors conclude that the PrP M129V polymorphism plays a modulatory role in the onset of WD, and they note that further studies are needed to determine whether the genotype of affected patients influences the severity of their symptoms and their long-term outcome during treatment.

Original article Merle U et al. (2006) Influence of homozygosity for methionine at codon 129 of the human prion gene on the onset of neurological and hepatic symptoms in Wilson disease. Arch Neurol 63: 982-985

\section{Hyperforin shows therapeutic potential in a rodent model of amyloidosis}

The formation of amyloid- $\beta$ peptide $(A \beta)$ fibrils in areas of the brain related to memory is an important event in the development of Alzheimer's disease. Studies in rodents have suggested that hyperforin isolated from Hypericum perforatum (St John's wort) might 